\title{
Intrinsic versus superrough anomalous scaling in spontaneous imbibition
}

\author{
M. Pradas* and A. Hernández-Machado \\ Departament d'Estructura i Constituents de la Matèria, Universitat de Barcelona, Avinguda Diagonal 647, E-08028 Barcelona, Spain
}

(Received 30 March 2006; revised manuscript received 13 July 2006; published 27 October 2006)

\begin{abstract}
We study spontaneous imbibition using a phase field model in a two-dimensional system with a dichotomic quenched noise. By imposing a constant pressure $\mu_{a}<0$ at the origin, we study the case when the interface advances at low velocities, obtaining the scaling exponents $z=3.0 \pm 0.1, \alpha=1.50 \pm 0.02$, and $\alpha_{l o c}=0.95 \pm 0.03$ within the intrinsic anomalous scaling scenario. These results are in quite good agreement with experimental data recently published. Likewise, when we increase the interface velocity, the resulting scaling exponents are $z=4.0 \pm 0.1, \alpha=1.25 \pm 0.02$, and $\alpha_{l o c}=0.95 \pm 0.03$. Moreover, we observe that the local properties of the interface change from a superrough to an intrinsic anomalous description when the contrast between the two values of the dichotomic noise is increased. From a linearized interface equation we can compute analytically the global scaling exponents which are comparable to the numerical results, introducing some properties of the quenched noise.
\end{abstract}

DOI: 10.1103/PhysRevE.74.041608

PACS number(s): 68.08.- $\mathrm{p}, 68.35 . \mathrm{Ct}$

\section{INTRODUCTION}

The growth of fluctuating interfaces in disordered media has been a subject of much interest in the last few years $[1,2]$. The study of the interface kinetic roughening is a fundamental problem in nonequilibrium statistical physics and has important applications in technological processes and material characterization. Examples of experimental studies are fracture surfaces [3,4], slow combustion fronts [5], and fluid-air interfaces in porous media [6-10]. The main common feature of the stable fluctuating interface $h(x, t)$ in such experiments is that it can be described in terms of scaling laws [11]. The basic assumption is to consider the FamilyVicsek hypothesis [12], which supposes a self-affine interface described by a growth exponent $\beta$, a roughness exponent $\alpha$, and a dynamic exponent $z$, with the scaling relation $\alpha=z \beta$. Although this scaling hypothesis is valid in a great variety of cases, different experiments $[4,8]$ and numerical models $[13,14]$ suggest a new scaling description, the socalled anomalous scaling, in the sense that the interface behaves in a not self-affine manner [15] and the local and global interface fluctuations are quite different so that a new local roughness exponent $\alpha_{l o c}$ has to be introduced. As pointed out by López [16], anomalous scaling appears due to the fact that the mean local slope of the interface $\langle\nabla h\rangle$ has a nontrivial dynamics. Furthermore, depending on the local exponent values, anomalous scaling can be classified in different groups such as the superrough or intrinsic anomalous scaling [17].

The advancement of a fluid into a porous medium has received much attention both experimentally and numerically $[1,2]$. It is important to remark that experiments can be carried out in two different ways: (a) forced-flow imbibition, where the invading fluid gets into the porous medium at a constant injection rate and (b) spontaneous imbibition, where the fluid advances into the medium due to the capillarity. In the present paper we focus on the spontaneous imbibition

*Electronic address: pradas@ecm.ub.es phenomenon (see Sec. II A), and we base our study on an experimental work that appeared recently [6] where an oil-air interface advances in a horizontal Hele-Shaw cell with a random gap spacing between both glass plates. Traction due to capillarity is so strong that an external negative pressure must be applied to have a very slow motion of the roughening front. With these experimental conditions, the scaling exponents obtained are $z=3, \alpha \simeq 2$, and $\alpha_{l o c} \simeq 1$ within the intrinsic anomalous scaling framework.

The first attempt to describe spontaneous imbibition numerically was proposed by Dubé et al. $[18,19]$ employing a phase field model [20-23]. The model is based on the introduction of a partial differential equation (model B in [24]) with a quenched noise and it includes the local conservation law of the liquid bulk mass. The resulting scaling exponents in the studied regime are $z=4, \alpha=1.25$, and $\alpha_{l o c} \simeq 1$ within the superrough anomalous scaling.

The objective of the present paper is to make a deeper study of the spontaneous imbibition using the phase field model. Our aim is to reproduce the experimental result [6] and explain the origin of the intrinsic anomalous scaling. It turns out that the velocity of the interface is a relevant parameter. The exponent $z=3$ indicates a global dynamics driven by capillary forces which occurs at low velocities. Intrinsic anomalous scaling has never numerically been observed in spontaneous imbibition and a dynamical growth with $z=3$ has only been observed previously in the forced imbibition case $[1,20,22]$. By increasing the interface velocity we recover the $z=4$ predicted by Dubé et al. [18]. In the same line, by increasing the capillary contrast of the quenched noise we can move from a superrough to an intrinsic anomalous scaling. Furthermore, the scaling properties of the quenched noise will be discussed to determine analytically the numerically obtained exponents.

The outline of the paper is as follows. In Sec. II we present both the analytical model to describe spontaneous imbibition and the numerical phase field model. In Sec. III we review the statistical properties of rough interfaces describing the anomalous scaling, and in Sec. IV we analyze and discuss the obtained numerical results. The final conclusions are given in Sec. V. 


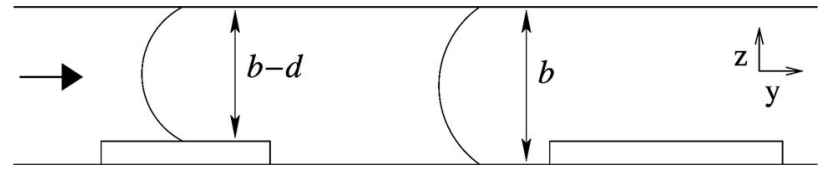

FIG. 1. Gap variation due to the quenched noise.

\section{THE MODEL}

\section{A. The spontaneous imbibition phenomenon}

Spontaneous imbibition consists in the advance of a viscous fluid of viscosity $\mu$ (like oil, water) in a porous medium with air, where motion is driven by capillarity. An important classical known result of this kind of motion is that the mean advancing front obeys Washburn's law [25], $H(t) \sim t^{1 / 2}$, thus spontaneous imbibition is a case with slowing-down dynamics.

Experimentally, a porous medium can be well-reproduced through the so-called Hele-Shaw cell, which consists of two horizontal glass plates of size $L_{x} \times L_{y}$, separated by a small gap $b$ in the $z$-direction, where a fiberglass substrate with copper squares is attached to the bottom plate (see Ref. [6]) producing a random gap spacing. A scheme of the meniscus curvature of the interface variation due to the quenched noise is depicted in Fig. 1.

The main effect of this random gap is to produce a random capillarity and permeability. However, in our specific case of spontaneous imbibition, where the motion is controlled solely by capillary forces and the interface advances at very low velocity, the fluctuations due to the permeability will be less important than those produced due to the random capillarity. Actually, it has been shown that the permeability term depends always on the interface velocity [20,26,27]. In this sense, we can suppose just the random capillarity as the main destabilizing term. Note that this approximation could not be valid in the forced-flow imbibition case, where the interface advances at constant velocity and thus the random permeability could become an important term, depending on the imposed velocity.

With this approximation in mind together with the fact that $b \ll L_{x}, L_{y}$, the equation of motion for the viscous fluid

$$
\nabla^{2} \boldsymbol{v}=\frac{1}{\mu} \nabla p
$$

is reduced to the two-dimensional (2D) Darcy's law:

$$
\overline{\boldsymbol{v}}(x, y)=-K \nabla p(x, y),
$$

where $\bar{v}$ represents the averaged velocity over the direction $z$. Since we considerer a constant permeability $K=b^{2} / 12 \mu$, we can impose the liquid conservation $[26], \boldsymbol{\nabla} \cdot \overline{\boldsymbol{v}}=0$, obtaining the Laplace equation for the pressure

$$
\nabla^{2} p=0
$$

The boundary conditions

$$
\left.\Delta p\right|_{\text {int }}=\sigma \kappa+\eta(x, h)
$$

$$
p(x, y=0)=P_{a}
$$

complete the equations to describe the spontaneous imbibition phenomenon. Equations (2)-(5) are the equations of the macroscopic model. Equation (4) is the local thermodynamic equilibrium condition at the interface, where $\sigma$ is the surface tension of the liquid, $\kappa$ is the local curvature of the interface, and $\eta(x, h)$ represents the random capillarity due to fluctuations in gap spacing, which it can be approached as $\eta(x, h)$ $\sim 2 \sigma \cos \theta / b$. We also assume that the contact angle $\theta$ does not vary.

On the other hand, Eq. (5) is the applied pressure at the origin of the cell and it is the main difference between the spontaneous imbibition and the forced-flow imbibition, since in the last case, a constant velocity is imposed.

\section{Washburn's law}

As a simple case of constant capillarity, $\eta(x, y)=\eta_{0}$, the problem is reduced to compute Eqs. (2) and (3) for the advance of a flat interface with the boundary conditions, Eqs. (4) and (5), where now $\kappa=0$; obtaining the exact solution

$$
\frac{\mathrm{d} H(t)}{\mathrm{d} t}=\frac{K\left(\eta_{0}+P_{a}\right)}{H(t)},
$$

where $H(t)$ is the position of the interface. Integrating the last expression one obtains the so-called Washburn's law

$$
H(t)=\sqrt{H_{0}^{2}+2 a t},
$$

where $a=K\left(\eta_{0}+P_{a}\right)$ and we have supposed an initial height of liquid $H_{0}$. Therefore, the expected behavior $H(t) \sim t^{1 / 2}$ is recovered after an initial transient regime.

\section{Linearized interface equation}

The general problem of a random field $\eta(x, y)$ is more complex to solve. The interface equation can be derived from Eqs. (3)-(5) using Green function analysis. In this case, the Green function $G\left(\boldsymbol{r}, \boldsymbol{r}^{\prime}\right)$ obeys $\nabla^{2} G\left(\boldsymbol{r}, \boldsymbol{r}^{\prime}\right)=\delta\left(\boldsymbol{r}-\boldsymbol{r}^{\prime}\right)$ evaluated in the semiplane $\Omega=\{x, 0 \leqslant y\}$ with Dirichlet boundary conditions. In order to get an expression for interface fluctuations, the Green equation

$$
\begin{aligned}
& \iint_{\Omega_{L}} d \boldsymbol{r}^{\prime}\left[p\left(\boldsymbol{r}^{\prime}\right) \nabla^{\prime 2} G\left(\boldsymbol{r}, \boldsymbol{r}^{\prime}\right)-G\left(\boldsymbol{r}, \boldsymbol{r}^{\prime}\right) \nabla^{\prime 2} p\left(\boldsymbol{r}^{\prime}\right)\right] \\
& =\int_{S_{L}} d \boldsymbol{s}^{\prime} \cdot p\left(s^{\prime}\right) \nabla^{\prime} G\left(s, s^{\prime}\right)-\int_{S_{L}} d \boldsymbol{s}^{\prime} \cdot G\left(s, s^{\prime}\right) \boldsymbol{\nabla}^{\prime} p\left(s^{\prime}\right)
\end{aligned}
$$

is integrated over the volume of the liquid $\Omega_{L}:\{x, 0 \leqslant y$ $\leqslant h(x, t)\}$, where $h(x, t)$ is the interface position, and the Darcy equation, Eq. (2), is imposed at the interface. Linearizing in small deviations of the height $h(x, t)$ around their mean value $H(t)$ in the Fourier space, one can obtain the linearized interface equation

$$
\dot{\tilde{h}}_{q}=-\sigma K|q| q^{2} \tilde{h}_{q}-\dot{H}(t)|q| \tilde{h}_{q}+K|q| \tilde{\eta}_{q},
$$

where we have supposed that correlations grow up slower in time than the position of the averaged interface over $x$, which 
lies in the condition $|q| H(t) \gg 1$. The noise term in Fourier space is taken as $\tilde{\eta}_{q}=\int d x e^{-i q x} \eta[x, h(x, t)]$. The zero mode of the interface position follows the Washburn equation, Eq. (6), in the form $\dot{H}(t)=a / H(t)$, where now, $a$ depends on the noise average $\langle\eta[x, h(x, t)]\rangle_{x}$ instead of $\eta_{0}$.

The linearized equation, Eq. (9), is the same equation obtained in Ref. [18] and contains two mechanisms to damp the interface fluctuations produced by the quenched noise $\widetilde{\eta}_{q}$. At small scales, the surface tension $\sigma$ controls the fluctuations introducing a dynamic exponent $z=3$. Actually, it is the regime observed experimentally in Ref. [6], indicating that a linear description is valid in that situation. The long scales are damped by the advancement of the front $\dot{H}(t)$. These two effects are separated by a dynamic crossover length:

$$
\xi_{\times}=2 \pi\left(\frac{\sigma K}{\dot{H}(t)}\right)^{1 / 2} .
$$

Since the mean height of the interface follows the Washburn law, this crossover length increases with time, $\xi_{\times} \simeq t^{1 / 4}$. The numerical results presented in Refs. [18,19] show that this crossover length acts as a cutoff for the fluctuations growth due to the interface being always asymptotically flat on length scales larger than $\xi_{\times}$. Thus if the velocity of the interface is large enough, the roughening of the interface is controlled by $\xi_{\times}$which gives rise to a dynamic exponent $z$ $=4$.

\section{B. The phase field model}

Our numerical approach is based on the phase field model, already introduced in Ref. [18], where a conserved field $\phi$ is used to represent the two phases of the problem: liquid/air with the equilibrium values $\phi_{e q}=+1 /-1$, respectively. The dynamics of the field is controlled by a continuity equation based on a time-dependent Ginzburg-Landau model with conserved order parameter (model B in [24]) $\partial \phi / \partial t$ $=\nabla M \nabla \mu$ where $\mu=\delta \mathcal{F} / \delta \phi$ is the chemical potential and the free energy is taken with the form $\mathcal{F}[\phi]=\int d \boldsymbol{r}[V(\phi)$ $\left.+(\epsilon \nabla \phi)^{2} / 2\right]$. A double well potential is also chosen with a linear random term, which destabilizes one of the phases and forces the interface to advance:

$$
V(\phi)=-\frac{1}{2} \phi^{2}+\frac{1}{4} \phi^{4}-\eta(\boldsymbol{r}) \phi .
$$

Here, the noise $\eta(\boldsymbol{r})$ plays the capillarity role. In our numerical integrations we use a dichotomic noise distributed in a 2D system with the values:

$$
\eta=\left\{\begin{array}{c}
\eta_{0} \\
\frac{\eta_{0}}{1-\eta_{A}} .
\end{array}\right.
$$

The noise is imposed in the same way as that in the experimental work in a Hele-Shaw cell [6], and it is distributed in such a way that the sites with $\eta_{0}$ occupy $65 \%$ of the whole system. Even though in such experiments capillarity arises as a $3 \mathrm{D}$ effect, we can relate our noise parameters with the

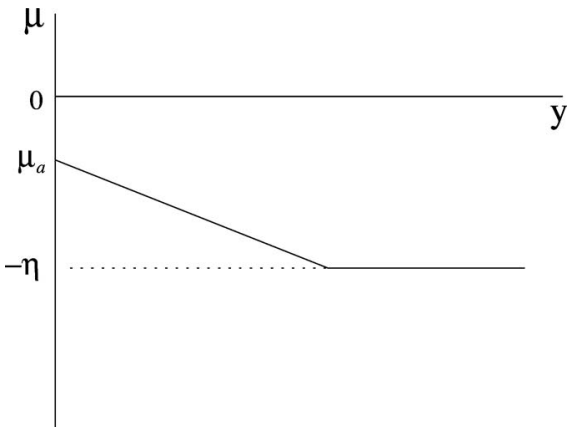

FIG. 2. Variation of the chemical potential in an arbitrary time along the $y$-direction. The velocity of the interface is related to $-\partial_{y} \mu$.

Hele-Shaw gap spacing $b$ as $\eta_{0} \sim 1 / b$ and $\eta_{A} \sim d / b$. So the $\eta_{A}$ parameter controls the contrast between both noise values whereas the $\eta_{0}$ corresponds to the capillarity of the cell with a fixed gap.

The resulting equation for the phase field reads

$$
\begin{aligned}
\frac{\partial \phi}{\partial t} & =\nabla M \nabla\left[V^{\prime}(\phi)-\epsilon^{2} \nabla^{2} \phi\right] \\
& =\nabla M \nabla\left[-\phi+\phi^{3}-\epsilon^{2} \nabla^{2} \phi-\eta(\boldsymbol{r})\right],
\end{aligned}
$$

where $M$ is a parameter which we take constant at the liquid phase $(\phi>0)$ and zero at the air phase $(\phi<0)$.

In order to recover the macroscopic results described by Eqs. (2)-(5), a matched asymptotic expansion in the sharp interface limit, $\epsilon \rightarrow 0$, is necessary. This expansion has been done in Ref. [28]. Although in that case the phase field is used to describe the Saffman-Taylor problem, a similar expansion is also valid in our case of a stable interface, with the identification of the phase field parameters as

$$
p=\phi_{e q} \mu_{1}, \quad K=\frac{M}{2 \phi_{e q}^{2}}, \quad \sigma=\frac{1}{2} \int d w\left(\frac{\partial \phi_{0}}{\partial w}\right)^{2},
$$

where $\mu_{1}$ is the first order in the $\epsilon$ term of the chemical potential in the asymptotic expansion and $\phi_{0}=-\phi_{e q} \tanh (w / \sqrt{2})$ is a kink solution of the phase field equation for a flat interface in equilibrium, and it corresponds to the zero order in the $\epsilon$ term of the order parameter. The variable $w$ is an inner coordinate of the interface, introduced in the expansion, and it is perpendicular to it at any point.

Since the chemical potential is equivalent to the pressure, it has to be fixed at the origin of our system to reproduce spontaneous imbibition, $\mu(x, y=0)=\mu_{a}$. In Fig. 2 there is plotted a scheme of the chemical potential variation along the $y$-direction. The imbalance created in the chemical potential by $\mu_{a}+\eta$ causes the interface to advance following the Washburn law, Eq. (7).

Throughout the paper we call applied pressure to the value $\mu_{a}$. We will also suppose an initial height of liquid $H_{0}$ to get a low initial velocity, Eq. (6). 


\section{SCALING OF ROUGH INTERFACES}

The statistical properties of a one-dimensional interface defined by a function $h(x, t)$ are usually described in terms of the fluctuations of $h$. The Family-Vicsek scaling hypothesis [12] ensures the existence of a lateral correlation length $\ell_{c}$ expected to increase as $\ell_{c} \sim t^{1 / z}$ until it reaches the system size $\ell_{c}=L$, which it defines a saturation time $t_{s} \sim L^{z}$. On the other hand, the vertical correlation length or interface global width, defined as $W(L, t)=\left\langle[h(x, t)-\bar{h}]^{2}\right\rangle^{1 / 2}$, increases as $W(L, t) \sim t^{\beta}$ for $t<t_{s}$ and becomes constant $W(L, t) \sim L^{\alpha}$ for $t \geqslant t_{s}$. Here, $\langle\cdot$.$\rangle denotes the average over different noise re-$ alizations and the overbar is a spatial average in the $x$ direction. Alternatively, one may study the correlations over a distance $\ell \ll L$ through the local width, $w(\ell, t)=\langle\langle[h(x, t)$ $\left.\left.\left.-\langle h\rangle_{\ell}\right]^{2}\right\rangle_{\ell}\right\rangle^{1 / 2}$, where $\langle\cdots\rangle_{\ell}$ denotes an average over $x$ in windows of size $\ell$. Since in the Family-Vicsek scenario the local scale grows in the same way as the global ones, the scaling behavior of the surface can be obtained by looking at the local width.

Another useful quantity also used to obtain the roughness exponent $\alpha$ is the power spectrum of the interface $S(k, t)$ $=\left\langle\tilde{h}_{k}(t) \tilde{h}_{-k}(t)\right\rangle$, where $\tilde{h}_{k}(t)$ is the interface Fourier transform. In the Family-Vicsek assumption it scales as

$$
S(k, t)=k^{-(2 \alpha+1)} s_{F V}\left(k t^{1 / z}\right),
$$

where $s_{F V}$ is the scaling function

$$
s_{F V}(u) \sim \begin{cases}\text { const } & \text { if } u \gg 1 \\ u^{2 \alpha+1} & \text { if } u \ll 1 .\end{cases}
$$

However, experimental results and several growth models have appeared in the last decade showing that global and local scales are not equivalent. It is the so-called anomalous scaling [14]. In this sense, a new scaling ansatz is needed to characterize both global and local growth.

This new scaling ansatz was proposed by Ramasco et al. [17] and it was a generalization of the Family-Vicseck scaling function, Eq. (16),

$$
S(k, t)=k^{-(2 \alpha+1)} s_{A}\left(k t^{1 / z}\right),
$$

where now the scaling function has the general form

$$
s_{A}(u) \sim \begin{cases}u^{2\left(\alpha-\alpha_{s}\right)} & \text { if } u \gg 1 \\ u^{2 \alpha+1} & \text { if } u \ll 1,\end{cases}
$$

$\alpha_{s}$ being the spectral roughness exponent. This scaling behavior of the power spectrum leads to the following dynamic scaling for the local width:

$$
w(\ell, t)=t^{\beta} g\left(\ell / t^{1 / z}\right),
$$

with the corresponding scaling function

$$
g(u) \sim \begin{cases}u^{\alpha_{l o c}} & \text { if } u \ll 1 \\ \text { const } & \text { if } u \gg 1,\end{cases}
$$

where $\alpha_{l o c}$ is the local rough exponent and it characterizes the roughness at small scales. One of the implications of the anomalous scaling is that the local width saturates when the system size saturates as well, i.e., at times $t_{s}$ and not at the

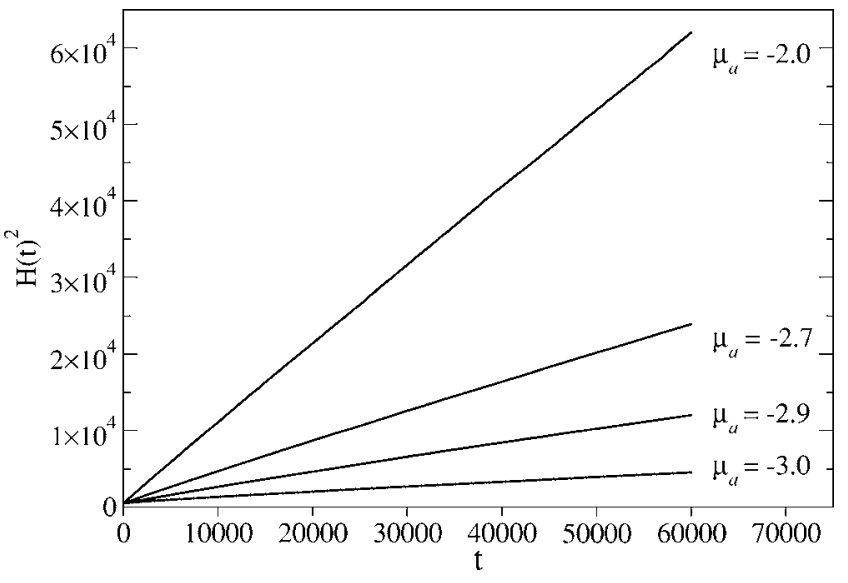

FIG. 3. Squared averaged interface height position, as a function of time, follows Washburn law for different applied pressure $\mu_{a}$.

local time $t_{\ell} \sim \ell^{z}$ as occurs in the Family-Vicsek scaling. There is an intermediate regime between $t_{\ell}$ and $t_{s}$ where the local width grows as $w(\ell, t) \sim t^{\beta^{*}}$ with $\beta^{*}=\beta-\alpha_{l o c} / z$.

Different types of scaling arise from the general scaling function, Eq. (17). For $\alpha_{s}<1$ it is always accomplished that $\alpha_{l o c}=\alpha_{s}$; in this case the Family-Vicsek scaling is achieved when $\alpha_{l o c}=\alpha$, and the intrinsic anomalous scaling appears when $\alpha>\alpha_{l o c}$. The main feature of such an anomalous scaling is the existence of a temporal shift in the power spectrum due to the difference between $\alpha_{l o c}$ and $\alpha$. On the other hand, for $\alpha_{s}>1$ it always occurs that $\alpha_{l o c}=1$ and the superrough anomalous scaling appears when $\alpha=\alpha_{s}$.

\section{NUMERICAL RESULTS}

The numerical study of the imbibition phenomenon is made by means of the integration of the phase field equation, Eq. (13), in a rectangular lattice of $\Delta x=1$ grid space, with periodic boundary conditions in the $x$ direction. We have also considered a smaller grid space $\Delta x=0.5$ and we reproduce identical results as we explain below. The interface position $h(x, t)$ is approached by a linear interpolation of the zero of the phase field, $\phi[x, h(x, t) ; t]=0$. In all of our numerical integrations we will suppose an initial height $H_{0}=20$ and the noise value $\eta_{0}=3.0$. Our study is focused to obtain the different scaling exponents and its dependence on the applied pressure, $\mu_{a}$, and on the contrast between noise values $\eta_{A}$.

\section{A. Variation in the applied pressure}

Different applied pressures at the origin have been studied. In all of them the Washburn law, Eq. (7), is wellaccomplished as we can see from Fig. 3. As a first case to analyze, we choose the numerical parameters $\eta_{A}=0.3$ and $\mu_{a}=-3.0$. In Fig. 4 there is plotted the temporal evolution of the interface. Note that the applied pressure has the same value in the module as the noise value $\eta_{0}$ and thus there are grid sites where the interface is locally pinned (Fig. 2). In this situation, the interface has a slow motion and advances in avalanches. 


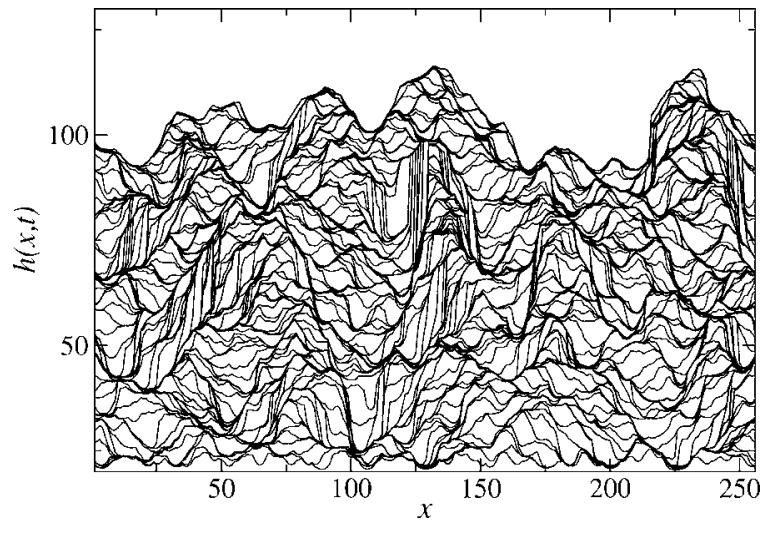

FIG. 4. Temporal evolution of the interface at equal time intervals. Numerical parameters are $\eta_{0}=3.0, \eta_{A}=0.3$, and $\mu_{a}=-3.0$.

Using the scaling concepts described in the last section, we do a statistical description of the interface fluctuations, Fig. 5. From the interface local width we obtain the global growth exponent $\beta=0.49 \pm 0.03$ and the local one $\beta^{*}$ $=0.20 \pm 0.04$, clearly different from 0 , indicating the existence of anomalous scaling. On the other hand, from the interface power spectrum we obtain the spectral roughness exponent $\alpha_{s}=1.0 \pm 0.1$. The temporal shift observed in the power spectrum indicates that $\alpha>\alpha_{s}$ and since $\alpha_{s}$ is not greater than 1, the relation $\alpha_{l o c}=\alpha_{s}$ should be accomplished [17]. Therefore the global roughness exponent $\alpha$ cannot be achieved directly from the power spectrum but it has to be obtained by means of a collapse of the interface local width function, Eq. (19). The result of the best collapse is shown in Fig. 5(b) from which we obtain that $\alpha=1.50 \pm 0.02$ and $z$ $=3.0 \pm 0.1$. This plot also shows a local roughness exponent of $\alpha_{l o c}=0.95 \pm 0.03$, which is very close to the value of the roughness exponent, $\alpha_{s}$. These sets of scaling exponents are compatible within the intrinsic anomalous scaling scenario, and they are clearly different from those obtained in Ref. [18].

Let us now increase the interface velocity by changing up the applied pressure, $\mu_{a}$. Following the same statistical procedure as before, we compute the whole set of scaling exponents for different values of $\mu_{a}>-3.0$. Figure 6 shows the global width $W(L, t)$ in each case. We observe a crossover from $\beta \simeq 0.5\left(\mu_{a}=-3.0\right)$ to $\beta \simeq 0.37\left(\mu_{a}=-2.7\right)$. In the specific case of $\mu_{a}=-2.7$, Fig. 7 , the different scaling exponents are $\alpha=1.5 \pm 0.02, \quad z=4.0 \pm 0.1, \quad \beta=0.37 \pm 0.03$, and $\alpha_{l o c}$ $=0.95 \pm 0.03$.

Finally, the results obtained in Ref. [18], $\alpha=1.25, z=4$, $\beta \simeq 0.32$, and $\alpha_{l o c} \simeq 1$, are recovered when we increase the applied pressure up to $\mu_{a}=-1.0$. So it is important to remark that decreasing the applied pressure produces three important effects: (1) the roughness exponent $\alpha$ changes from 1.25 to 1.5, , (2) the interface local growth changes from a superrough to an intrinsic anomalous scaling description, and (3) the dynamic exponent $z=3$ obtained in experimental work is attained when the interface advances at low velocities. In Table I we can see the different scaling exponents computed for different values of $\mu_{a}$.

The meaning of large and low velocities has to be understood as follows. The interface advances at low velocity
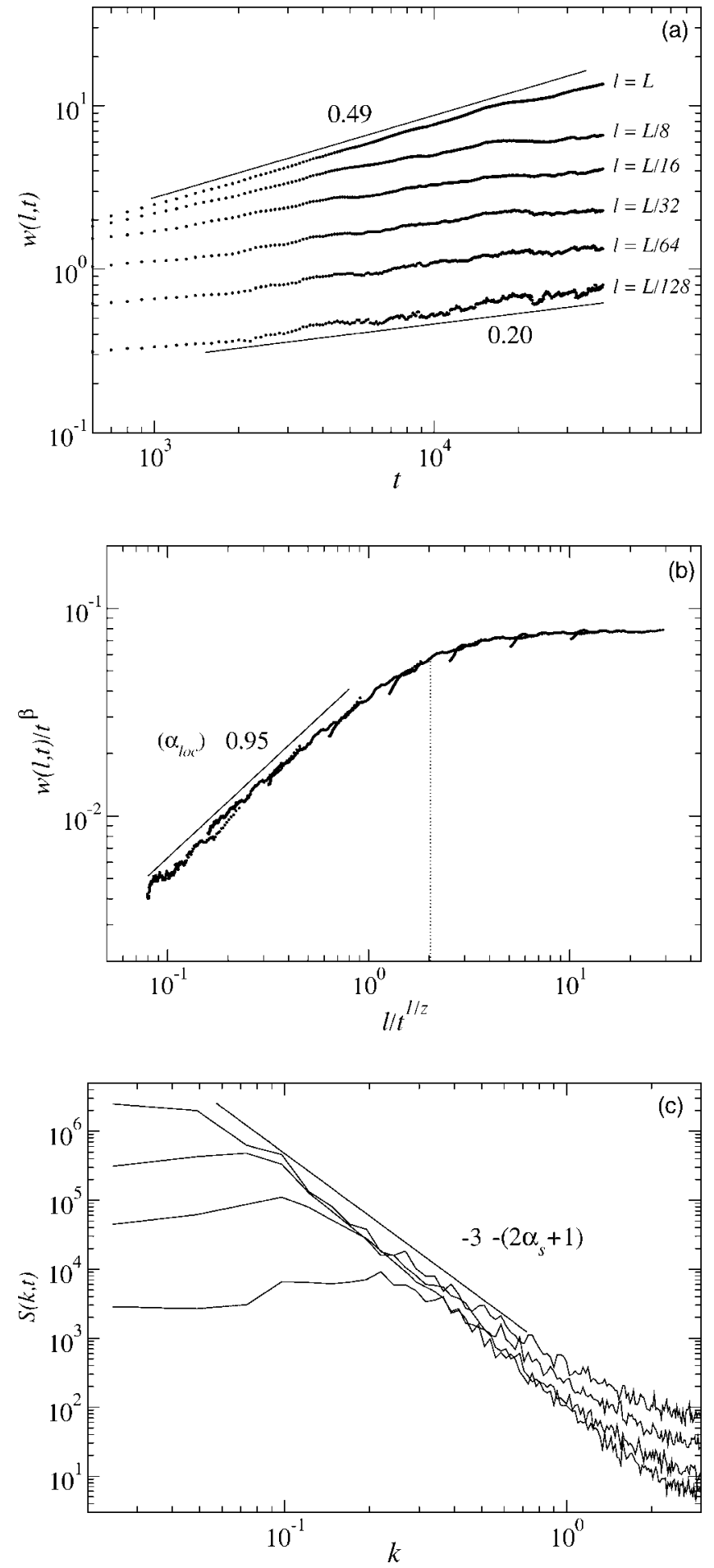

FIG. 5. Statistical description of interfaces when $\mu_{a}=-3.0$ and $\eta_{A}=3.0$ : (a) Log-log plot of the interfacial width as a function of time in different windows sizes $\ell$. The data fit shows a growth exponent $\beta=0.49 \pm 0.03$ and the local one $\beta^{*}=0.20 \pm 0.04$. (b) Collapse of the interface local width function, $w(\ell, t)$, which is scaled by $t^{\beta}$ vs $\ell / t^{\beta / \alpha}$, where $\alpha=1.50 \pm 0.02$, and then $z=3.0 \pm 0.1$ follows from $z=\alpha / \beta$. The picture also shows a local roughness exponent $\alpha_{l o c}=0.95 \pm 0.03$. (c) Power spectra of the interface $h(x, t)$ at different times. It shows that $\alpha_{s}=1.0 \pm 0.1$. 


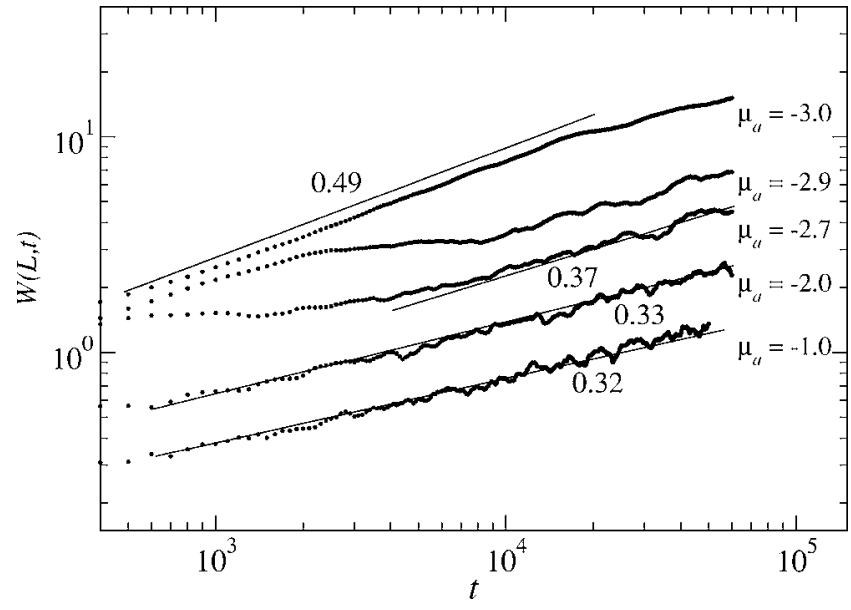

FIG. 6. Computed global width $W(L, t)$ by changing the applied pressure $\mu_{a}$. The lines show a fit data which allows us to obtain the $\beta$ exponent.

when the pinning effects become important and the crossover length is large enough to observe an initial regime described by $z=3$. Therefore, in this sense, we call low velocities the cases of $\mu_{a}<-2.0$ and large velocities the other ones, $\mu_{a} \geqslant$ -2.0 .

Concerning the low velocities regime, there is some numerical inaccuracy in the determination of the interface position due to the slow advance of the interface. In order to check our numerical results, we have performed the case of $\mu_{a}=-3.0$ with a smaller grid space. We have used $\Delta x=0.5$ and no difference between the case of $\Delta x=1.0$ has been observed.

\section{The crossover and correlation length}

So far we have seen that an initial regime, dominated by the surface tension term of Eq. (9), can be observed if the interface velocity is low enough. The point is that the correlation length $\ell_{c}=A t^{1 / z}$ is smaller than the crossover length $\xi_{\times}$ during the time interval studied. In order to check it, we are interested in estimating the crossover and correlation length. First, we suppose that in the case of $\mu_{a}=-2.7$, in the regime of $z=4$, the crossover length, $\xi_{\times}=A t^{1 / 4}$, acts effectively as the correlation length as Dubé et al. have pointed out [18], where the constant $A$ can be approached from the inflection point of the width collapse, Fig. $7(\mathrm{~b})$, being $A \simeq 2.5$. On the other hand we know that the crossover length has the expression $\xi_{\times}=C(2 / a)^{1 / 4} t^{1 / 4}$, obtained from Eq. (10) in the limit $H_{0}^{2}<2 a t$, where $a$ follows from Washburn law, Eq. (7). With these two expression we can obtain the constant $C \simeq 1.4$, which allows us to evaluate the crossover length in its full expression, Eq. (10), when $\mu_{a}=-3.0$. In such case the correlation length grows up as $\ell_{c}=2 t^{1 / 3}$, where now the parameter 2 has been estimated from the width collapse in Fig. 5(b). These results are depicted in Fig. 8, where we can observe that during the studied time interval, the condition $\xi_{\times}>\ell_{c}$ is accomplished, and thus the obtained dynamical exponent $z$ $=3$ is fully justified.

It should be noted from Eq. (7) that the crossover length takes a certain time $\tau=H_{0}^{2} / 2 a$ to reach its asymptotic form
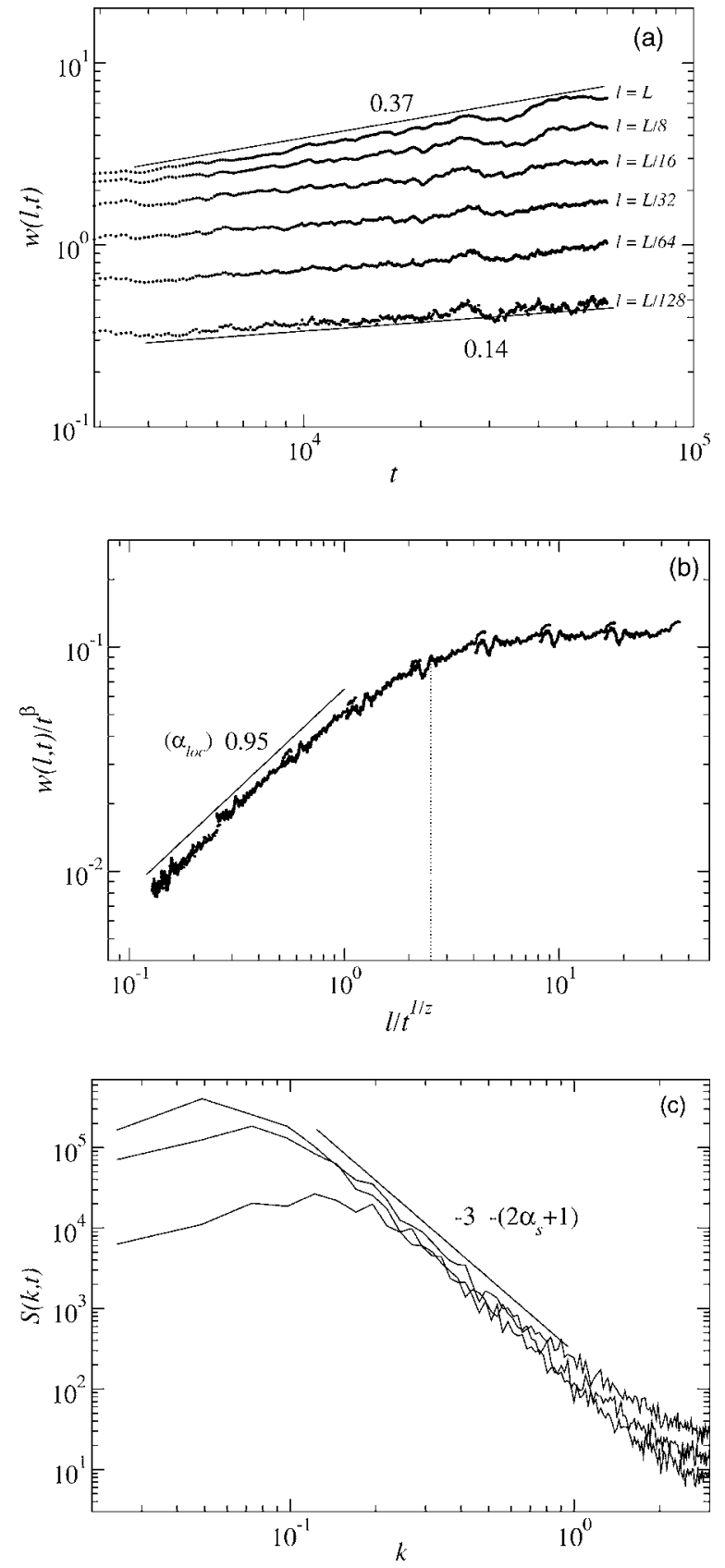

FIG. 7. Statistical description of interfaces when $\mu_{a}=-2.7$ and $\eta_{A}=3.0$ : (a) Log-log plot of the interfacial width as a function of time in different windows sizes $\ell$. The data fit shows a growth exponent $\beta=0.37 \pm 0.03$ and the local one $\beta^{*}=0.14 \pm 0.04$. (b) Collapse of the interface local width function, $w(\ell, t)$, which is scaled by $t^{\beta}$ vs $\ell / t^{\beta / \alpha}$, where $\alpha=1.50 \pm 0.02$, and then $z=4.0 \pm 0.1$ follows from $z=\alpha / \beta$. The picture also shows a local roughness exponent $\alpha_{l o c}=0.95 \pm 0.03$. (c) Power spectra of the interface $h(x, t)$ at different times. It shows that $\alpha_{s}=1.0 \pm 0.1$.

$\xi_{\times} \simeq t^{1 / 4}$. It could produce some effect on the interface scaling as long as $t_{\times} \leqslant \tau$, where $t_{\times}$is defined as the time when $\ell_{c}=\xi_{\times}$. As we can see in Fig. 6 , in both cases of $\mu_{a}=-2.9$ and $\mu_{a}=-2.7$, there is a regime, during the transition from $z=3$ to $z=4$, where the interface global width does not increase. Computing the parameter $a$ from a fit of the Washburn law, it turns out that $\tau \sim 2 \times 10^{3}$ and $10^{3}$ for $\mu_{a}=-2.9$ 
TABLE I. Scaling exponents obtained when the applied pressure $\mu_{a}$ decreases. The contrast of the noise values is kept constant $\eta_{A}$ $=0.3$.

\begin{tabular}{ccccccc}
\hline \hline$\mu_{a}$ & $\alpha$ & $z$ & $\beta$ & $\beta^{*}$ & $\alpha_{s}$ & Scaling \\
\hline-1.0 & 1.25 & 4 & 0.32 & 0.10 & 1.25 & S-R \\
-2.0 & 1.34 & 4 & 0.33 & 0.12 & 0.9 & I-A \\
-2.7 & 1.5 & 4 & 0.37 & 0.14 & 0.9 & I-A \\
-3.0 & 1.5 & 3 & 0.49 & 0.20 & 0.9 & I-A \\
\hline \hline
\end{tabular}

and -2.7 , respectively, indicating that $\tau \sim t_{\times}$in both cases. For larger values of the applied pressure this effect occurs in an initial stage of the interface growth and it is not observed.

An interesting point to comment on from these numerical results is that the roughness exponent $\alpha$ does not change its value when the correlation length catches up to the crossover length, that is, the growth exponent $\beta$ varies accordingly with the change of the dynamic exponent from $z=3$ to $z=4$ keeping constant the $\alpha$ value (see Fig. 6). Therefore it lead us to think that the crossover length does not change the scaling properties of the interface except for the dynamic exponent $z$. It can be very useful to extract information from the linear Eq. (9). Since the initial interface velocity has a finite value greater than zero, the condition $t_{\times}>0$ is always accomplished and therefore there will be always an initial regime where fluctuations are dominated by the surface tension. Note that this initial regime could be very short for a large initial velocity of the interface. Hence we can suppose that the scaling exponents $\alpha$ and $\alpha_{\text {loc }}$, observed when the crossover length is controlling the fluctuations growth, are the same as those corresponding to the initial regime described by $z=3$.

\section{Intrinsic anomalous scaling in the low velocity regime}

Our numerical results show the influence of the applied pressure on the scaling exponents. We have seen that the intrinsic anomalous scaling appears when we decrease the applied pressure. Actually, the large local slopes, which appear when the interface velocity is decreased, cause that the

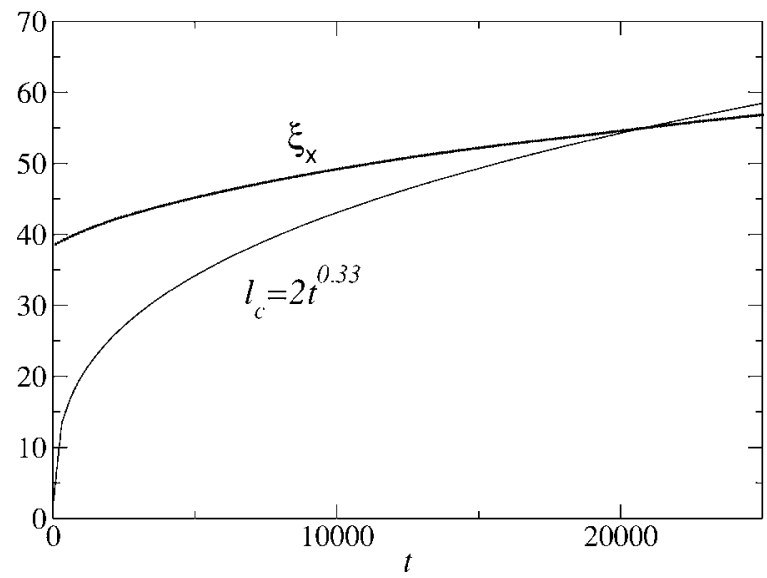

FIG. 8. Estimation of the crossover and correlation length when the dynamic exponent $z=3$ is observed.
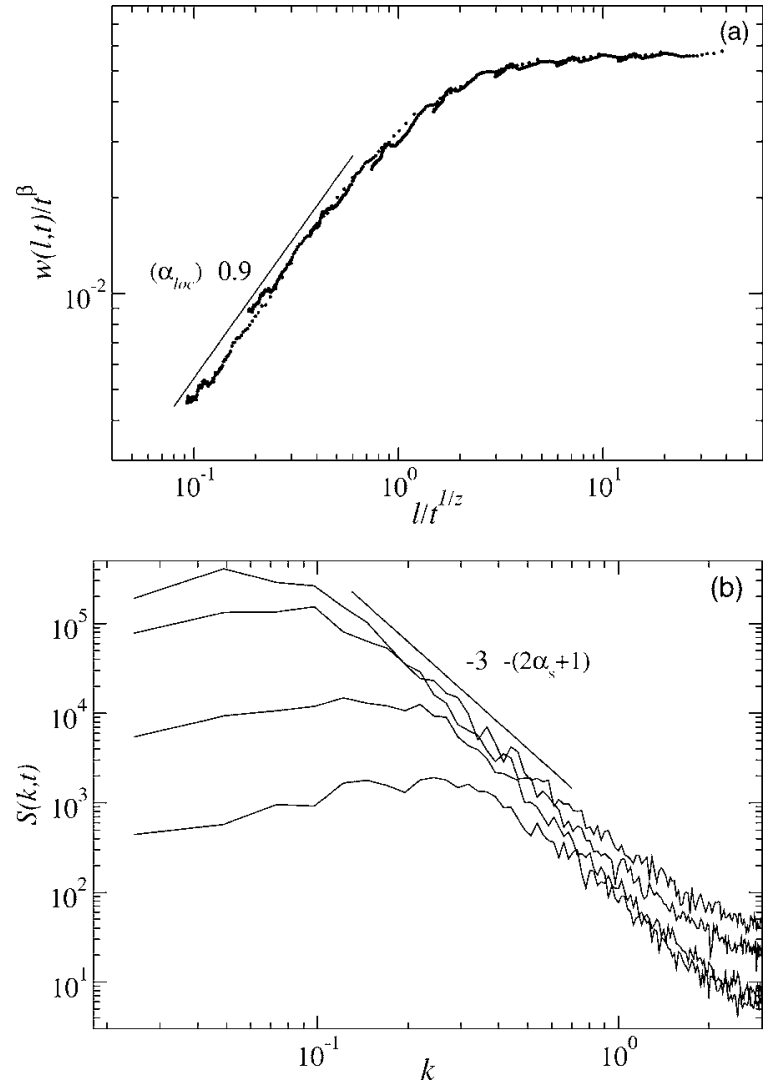

FIG. 9. Statistical description of interfaces described by Eq. (13) with a Gaussian noise distributed at $100 \%$ of the system with mean $\langle\eta\rangle=3.0$ and strength $\Delta \eta=0.6$. The applied pressure is $\mu_{a}=-2.5$. (a) Collapse of the interface local width function, $w(\ell, t)$, which is scaled by $t^{\beta}$ vs $\ell / t^{\beta / \alpha}$, where $\alpha=1.51 \pm 0.02, \beta=0.51 \pm 0.03$, and then $z=3.0 \pm 0.1$ follows from $z=\alpha / \beta$. The picture also shows a local roughness exponent $\alpha_{l o c}=0.9 \pm 0.03$. (c) Power spectra of the interface $h(x, t)$ at different times. It shows that $\alpha_{s}=1.0 \pm 0.1$.

scaling properties of the interface follow an intrinsic anomalous description.

In order to approach analytically the global exponents in the case of low velocities we should know the scaling properties of the noise. Likewise, the fact that the interface is locally pinned may introduce some difficulties on the quenched noise and we cannot know a priori how the noise scales. However, the roughness exponent $\alpha=1.5$ seems to indicate that the interface feels an effective white columnar noise, that is $\eta(x)$ with correlations $\left\langle\eta(x) \eta\left(x^{\prime}\right)\right\rangle \simeq \delta\left(x-x^{\prime}\right)$. Indeed, if we rescale the linearized interface equation, Eq. (9), by

$$
x \rightarrow b x, \quad h \rightarrow b^{\alpha} h, \quad t \rightarrow b^{z} t,
$$

the noise scales then as

$$
\eta(x) \rightarrow b^{-1 / 2} \eta(x)
$$

and the roughness exponent we obtain is $\alpha=1.5$. This roughness exponent is the same value obtained in the local quenched Edward-Wilkinson (QEW) model with columnar noise [29]. 


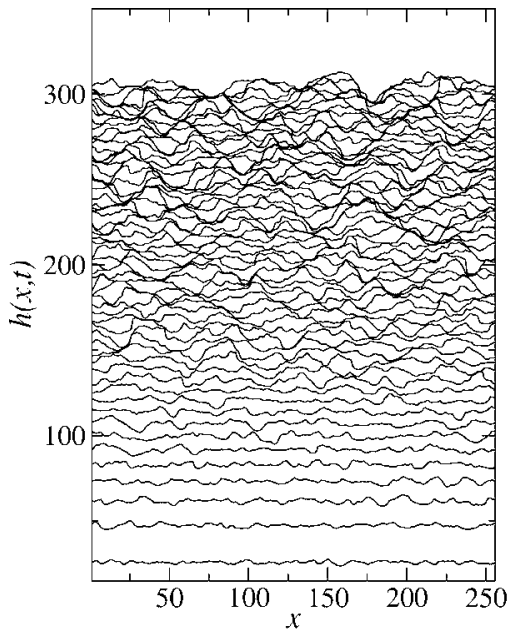

(a) Interfaces described by the super-rough anomalous scaling

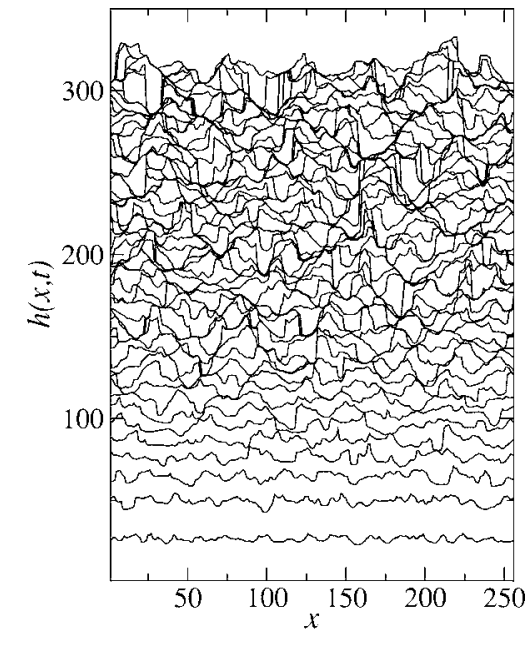

(b) Interfaces described by the intrinsic anomalous scaling
It should be pointed out that this assumption of columnar noise is related to the number of sites where the interface is pinned; note that the fractions of the interface which are locally pinned increases as the applied pressure decreases up to values lower than $\mu_{a}<-3.0$ where the interface does not advance at all. So in this sense, the roughness exponent $\alpha$ $=1.5$ is observed when the interface advances near a critical situation of pinning. These results agree with the hypothesis of Parisi [30] who suggest that near the pinning transition, both QEW and quenched Kardar-Parisi-Zhang (QKPZ) models follow the same universality class than in the QEW model with a columnar noise $\eta(x)$. However, we have to remark that only the roughness exponent $\alpha=1.5$ obtained in our model adjusts with such a universality class.

On the other hand, when the applied pressure is increased and therefore we move further away from the critical situation we recover the results of $\alpha=1.25$ corresponding to the universality class of the local QEW model with quenched noise $\eta(x, h)[1]$. In this sense, the other observed roughness exponent $\alpha \simeq 1.34$ can be understood as an intermediate regime between the value $\alpha=1.25$ and $\alpha=1.5$ when the applied pressure is decreased. Likewise, in the next section we will discuss an analytical approach which describes the quenched noise scaling in the high velocity regime, when the values $\alpha=1.25$ and 1.33 are observed.

In order to check the validity of these numerical results, we have performed the simulations with another kind of noise. We have used a Gaussian field distributed at $100 \%$ of the system with mean $\langle\eta\rangle=3.0$ and strength $\Delta \eta=0.6$. When the applied pressure is $\mu_{a}=-2.5$, the interface advances at low velocities and we recover the same results of $\alpha=1.5$ and $z=3$ within the intrinsic anomalous scaling, Fig 9. Therefore the columnar noise assumption is also correct when we use a different noise distribution.

Comparing to the experimental results, our $\alpha=1.5$ differs from the value $\alpha=2.0$ obtained in Ref. [6]. However, the other independent exponents, $\alpha_{l o c}$ and $z$ are in good agreement. This discrepancy in the $\alpha$ exponent could be attributed to a very low velocity of the experimental interface, not attained in the numerical integration of the phase field, intro- ducing a different property on the scaling of the quenched noise; however, it remains as an open question.

\section{B. Variation in the capillary contrast}

Another interesting issue to study is what happens when we increase the contrast between the two values of the noise keeping constant the applied pressure. Actually, it is similar to increasing the strongness of the capillarity of the HeleShaw cell and a similar study was carried out experimentally by Soriano et al. [9]. So in this sense, we are interested in studying the change of the scaling exponents when we increase the parameter $\eta_{A}$. Our starting point is the known results of superrough interfaces with $\alpha=1.25$ and $z=4 \mathrm{ob}-$ tained in the case of $\mu_{0}=-1.0$ and $\eta_{A}=0.3$. In Fig. 10 we can see two different interface profiles with two different capillary contrasts, $\eta_{A}=0.3$ and $\eta_{A}=0.5$, respectively, where we observe the morphological difference between both interfaces. While in the former case the interfaces are characterized by a smooth profile, large local slopes appear when the capillary contrast is increased. Note that due to numerical limitations, the capillary contrast is not able to be too large. Actually, for values $\eta_{A}>0.6$ we have observed that the interface becomes unstable and a clear statistical description of the interface fluctuations cannot be performed.

The different scaling exponents for the case of $\eta_{A}=0.5$ are computed in Fig. 11 obtaining: $\alpha=1.33 \pm 0.03, z=4.0 \pm 0.1$, $\beta=0.33 \pm 0.03, \beta^{*}=0.16 \pm 0.04$, and $\alpha_{l o c}=0.70 \pm 0.03$, which are compatible within the intrinsic anomalous scaling framework. In Table II we can realize how the scaling exponents change by varying the noise contrast. While the global exponents $(\alpha, \beta$, and $z)$ are quite similar in both cases, the interface local behavior changes from a superrough to an intrinsic anomalous description.

If we use a Gaussian noise instead of a dichotomic noise as in the previous section, we obtain a similar variation in the local scaling properties of the interface fluctuations. It turns out that increasing the strength of the Gaussian noise, $\Delta \eta$, an intrinsic anomalous scaling of the interface fluctuations is observed. Therefore there seems to be a relation between the 

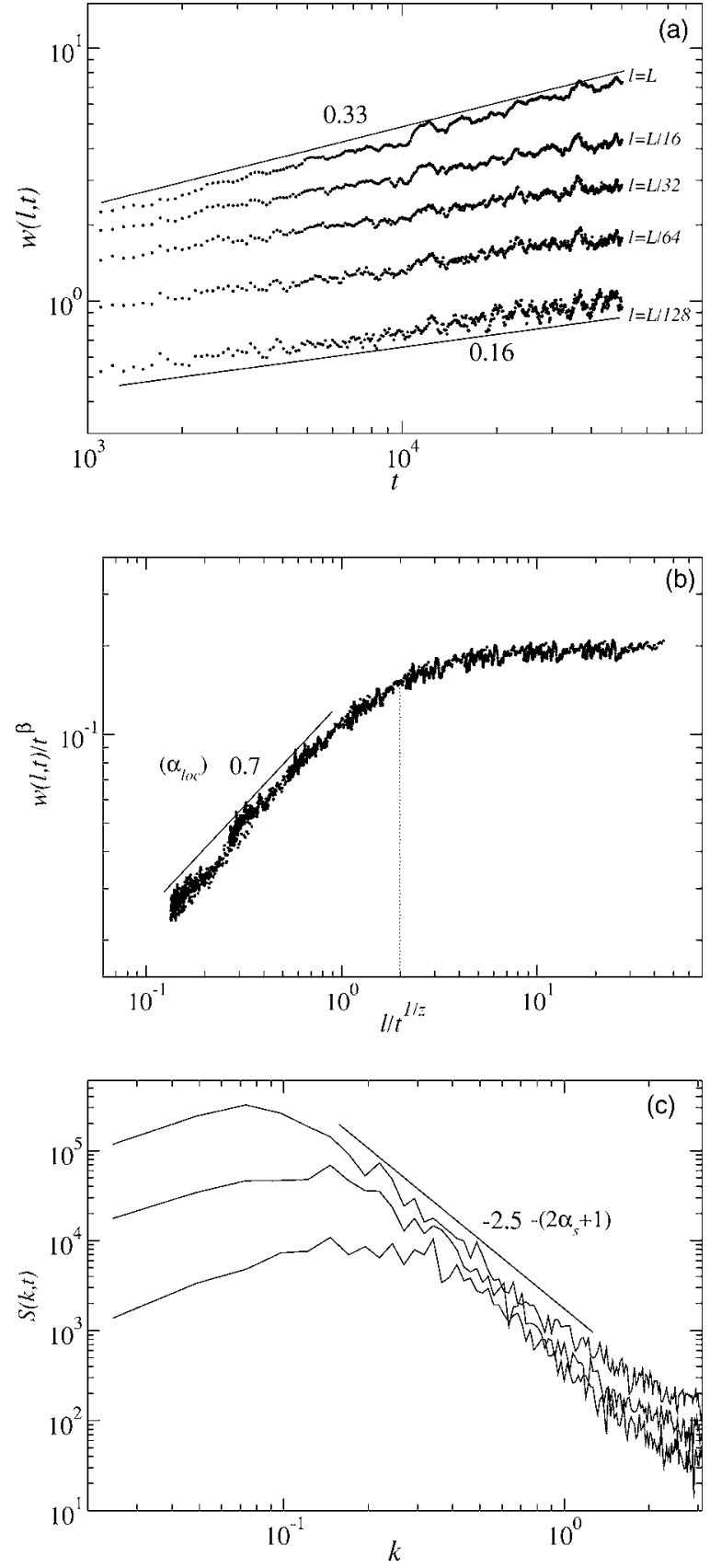

FIG. 11. Statistical description of the interface with an increased capillary contrast, $\eta_{A}=0.5$ : (a) Log-log plot of the interfacial width as a function of time in different windows sizes $\ell$. The data fit shows a growth exponent $\beta=0.33 \pm 0.03$ and the local one $\beta^{*}$ $=0.16 \pm 0.04$. (b) Collapse of the interface local width function, $w(\ell, t)$, which is scaled by $t^{\beta}$ vs $\ell / t^{\beta / \alpha}$, where $\alpha=1.33 \pm 0.03$, and then $z=4.0 \pm 0.1$ follows from $z=\alpha / \beta$. The picture also shows a local roughness exponent $\alpha_{l o c}=0.70 \pm 0.03$. (c) Power spectra of the interface $h(x, t)$ at different times. It shows that $\alpha_{s}=0.75 \pm 0.05$.

capillary contrast of the dichotomic noise and the strength of the Gaussian field. Likewise, Laurila et al. [22] have observed a slight dependence of the strength of Gaussian noise on the roughness exponent $\alpha$ in a similar way as our results of Table II when we change the capillary contrast of the dichotomic noise.
TABLE II. Scaling exponents for two different capillary contrast, $\eta_{A}$, with an applied pressure $\mu_{a}=-1.0$.

\begin{tabular}{ccccccc}
\hline \hline$\eta_{A}$ & $\alpha$ & $z$ & $\beta$ & $\beta^{*}$ & $\alpha_{s}$ & Scaling \\
\hline 0.3 & 1.25 & 4 & 0.32 & 0.10 & 1.25 & S-R \\
0.5 & 1.33 & 4 & 0.33 & 0.16 & 0.65 & I-A \\
\hline \hline
\end{tabular}

\section{Scaling properties of the quenched noise}

Our objective now is to try to determine analytically the global scaling exponents observed in each case of the studied capillary contrast. While the dynamic exponent $z$ can be well understood from the different terms of the linear equation, Eq. (9), the roughness exponent $\alpha$ involves the knowledge of the scaling of the noise and it can be a more difficult task.

First, we assume the general form for the noise correlation

$$
\left\langle\eta(x, h) \eta\left(x^{\prime}, h^{\prime}\right)\right\rangle \simeq f\left(x, x^{\prime}\right) g\left(h, h^{\prime}\right) .
$$

In order to describe the scaling of the noise, we are interested in finding out the expression for the functions $f\left(x, x^{\prime}\right)$ and $g\left(x, x^{\prime}\right)$. To do it, we first focus on the case of $\eta_{A}=0.5$ [Fig. 10(b)]. In this situation, an important feature of the interface velocity is that it has a great increase when the interface crosses through the sites where the noise takes the largest value of Eq. (12). In some way, the interface seems to avoid these sites and it prefers to be where the noise is lower, that is $\eta=\eta_{0}$. It leads one to think that there has to be a length $\lambda_{c}$ below which the noise has a constant value. Since there is no imposed velocity but the interface moves only due to the capillarity of the media, this length $\lambda_{c}\left(t, \eta_{A}\right)$ is expected to increase with time and with the capillary contrast. An interpretation of $\lambda_{c}$ can be seen in Fig. 12, where the values of the quenched noise $\eta(x, h)$ in a fixed interface $h\left(x, t_{f}\right)$ are plotted, $t_{f}$ being an arbitrary chosen time. Note that $\lambda_{c}$ has to be understood as an average length in such a way that we can suppose the function $f\left(x, x^{\prime}\right) \simeq$ const for $\left|x-x^{\prime}\right|<\lambda_{c}$ and $f\left(x, x^{\prime}\right) \simeq \delta\left(x-x^{\prime}\right)$ for $\left|x-x^{\prime}\right|>\lambda_{c}$.

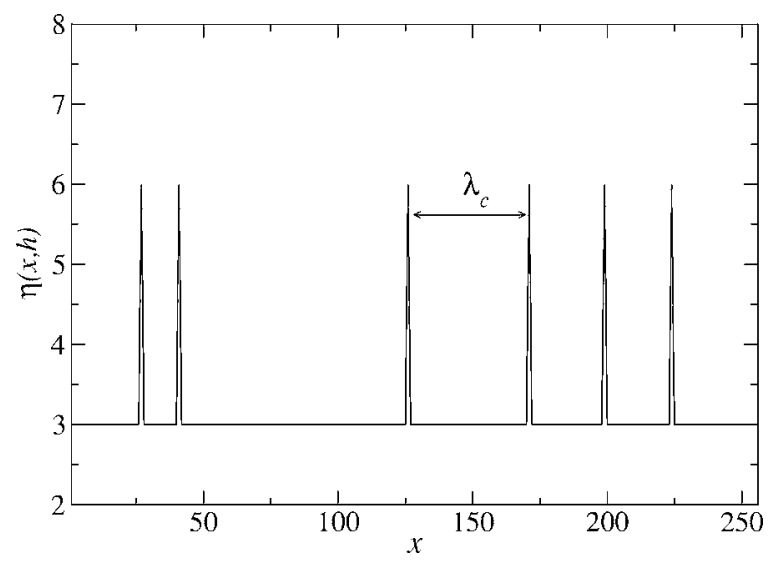

FIG. 12. Dichotomic noise, $\eta(x, h)$, evaluated in a fixed interface $h\left(x, t_{f}\right)$ for each $x$ in the case of the capillary contrast $\eta_{A}$ $=0.5$. $\lambda_{c}$ represents an averaged length where the noise takes a constant value. 


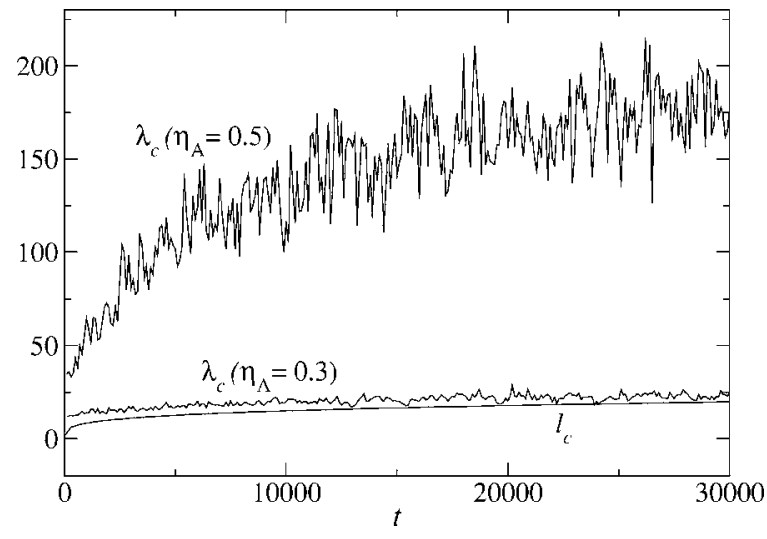

FIG. 13. The length $\lambda_{c}$ of the quenched noise is always greater than interface correlation length $\ell_{c}$ in both cases of $\eta_{A}=0.3$ and 0.5 .

Since the only length scale characterizing the fluctuations growth in the $x$ direction is the correlation length $\ell_{c}$, two different scalings for the noise emerge depending on if $\ell_{c}$ $>\lambda_{c}$ or $\ell_{c}<\lambda_{c}$. In Fig. 13 there is plotted the length $\lambda_{c}$ in the two cases of capillary contrast, $\eta_{A}=0.3$ and $\eta_{A}=0.5$, compared with the interface correlation length $\ell_{c} \simeq t^{1 / 4}$. We can see that in both cases $\lambda_{c}>\ell_{c}$ and thus we can take the correlation of the noise along the $x$-direction as a constant.

Concerning the expression for the function $g\left(h, h^{\prime}\right)$, we will suppose the simplest case of $g\left(h, h^{\prime}\right)=\delta\left(h-h^{\prime}\right)$. However, note that two different kinds of scaling emerge depending on if the quenched noise is evaluated on the interface $\eta(x, h)$ or on the mean value of the interface, $\eta(x, h)$ $\simeq \eta[x, H(t)]$. Therefore we conclude that the quenched noise has to scale as

$$
\begin{gathered}
\eta(x, h) \rightarrow b^{0} b^{-\alpha / 2} \eta(x, h), \\
\eta(x, H) \rightarrow b^{0} b^{-z / 4} \eta(x, H),
\end{gathered}
$$

when we rescale it by Eq. (21). The term with $b^{0}$ comes from the constant function $f\left(x, x^{\prime}\right)$. We have also assumed the Washburn law $H(t) \simeq t^{1 / 2}$.

It should be noted that these noise scalings are not valid when the interface movement is near a critical pinning situation (as in the previous section where $\mu_{a} \sim-3.0$ ) since in such a case there is an important number of sites where the interface is locally pinned and the effects of a columnar noise, Eq. (22), become important.

\section{Global scaling exponents}

Once we know how the noise scales, we can compute analytically the global scaling exponents in the studied cases of capillary contrast, $\eta_{A}=0.3$ and 0.5 . We do a scaling analysis of the linear equation, Eq. (9), taking into account only the surface tension term. Imposing the noise scaling as Eq. (24), we obtain the exponent $\alpha=1.33$, which is in accordance to the case of $\eta_{A}=0.5$. On the other hand, if we suppose that noise scales as Eq. (25), the exponent is then $\alpha=1.25$, which adjusts the numerical value obtained in Ref. [18] and in our case of $\eta_{A}=0.3$. Note that the $\alpha=1.33$ observed when $\eta_{A}$ $=0.5$ is the same as the value obtained in the Sec. IV A, in the case of $\mu_{a}=-2.0$ (see Table I), indicating that, in that case, the scaling of the noise has to follow also Eq. (24). Therefore we analytically obtain the roughness exponents in the high velocity regime when we vary either the capillary contrast or the applied pressure.

Eventually, since the crossover length controls the interface fluctuations in both cases, we conclude that the dynamic exponent has to be $z=4$, and then we achieve the whole set of global scaling exponents described in Table II.

\section{CONCLUSIONS}

We have presented numerical results of spontaneous imbibition using a phase field model. Our study has been focused on the dependence of the scaling exponents when we vary either the applied pressure imposed at the origin of the system or the capillary contrast of the dichotomic quenched noise.

By decreasing the applied pressure, we have got interfaces advancing at low velocities. In this situation of spontaneous imbibition, our results are in good agreement with the experimental work made in Ref. [6]. Indeed, our numerical study confirms that there is a regime of low velocities where the growth of the interface fluctuations is controlled by the surface tension of the interface, following $\ell_{c} \simeq t^{1 / 3}$. We have also observed that the cutoff crossover length, $\xi_{x}$, acts as an effective correlation length when the initial interface velocity is higher, accordingly to the results of Dubé et al. [18].

On the other hand, our results show that the local scaling properties of the interface change as we decrease the applied pressure. More precisely, we obtain an intrinsic anomalous description at the regime of low velocities, where the interface is locally pinned. In addition, the roughness exponent we observe in such a situation is $\alpha=1.5$ which is the same value obtained in the local QEW model with a columnar noise, $\eta(x)$.

It is also important to remark that the intrinsic anomalous scaling is not related to the low velocity of the interface but to the capillary contrast of the noise. Indeed, when we increase the contrast between both noise values keeping constant the applied pressure, the local fluctuations description of the interface changes from a superrough to an intrinsic anomalous scaling, indicating the existence of a direct relation of the capillary contrast and the intrinsic anomalous scaling, which had been observed experimentally in Ref. [9]. We have also introduced some scaling properties of the dichotomic quenched noise which have allowed us to determine analytically the obtained numerical scaling exponents.

\section{ACKNOWLEDGMENTS}

Fruitful discussions with J.M. López, M.A. Rodríguez, J. Ortín, R. Planet, and A.M. Lacasta are acknowledged. The research has received financial support from Project BFM2003-07749-C05. M.P. was supported by MEC, Spain. 
[1] M. Alava, M. Dubé, and M. Rost, Adv. Phys. 53, 83 (2004).

[2] T. Ala-Nissila, S. Majaniemi, and K. Elder, Lect. Notes Phys. 640, 357 (2004).

[3] E. Bouchard, J. Phys.: Condens. Matter 9, 4319 (1997).

[4] J. M. López and J. Schimttbuhl, Phys. Rev. E 57, 6405 (1998).

[5] J. Maunuksela, M. Myllys, O.-P. Kähkönen, J. Timonen, N. Provatas, M. J. Alava, and T. Ala-Nissila, Phys. Rev. Lett. 79, 1515 (1997).

[6] J. Soriano, A. Mercier, R. Planet, A. Hernández-Machado, M. A. Rodríguez, and J. Ortín, Phys. Rev. Lett. 95, 104501 (2005).

[7] J. Soriano, A. Hernández-Machado, and J. Ortín, Phys. Rev. E 66, 031603 (2002).

[8] J. Soriano, J. J. Ramasco, M. A. Rodríguez, A. HernándezMachado, and J. Ortín, Phys. Rev. Lett. 89, 026102 (2002).

[9] J. Soriano, A. Hernández-Machado, and J. Ortín, Phys. Rev. E 67, 056308 (2003).

[10] D. Geromichalos, F. Mugele, and S. Herminghaus, Phys. Rev. Lett. 89, 104503 (2002).

[11] A.-L. Barabási and H. E. Stanley, Fractal Concepts in Surface Growth (Cambridge University Press, Cambridge, England, 1995).

[12] F. Family and T. Vicsek, J. Phys. A 18, L75 (1985).

[13] H. Leschhorn and L.-H. Tang, Phys. Rev. Lett. 70, 2973 (1993).

[14] J. M. López, M. A. Rodríguez, and R. Cuerno, Physica A 246, 329 (1997).

[15] J. M. López and M. A. Rodríguez, Phys. Rev. E 54, R2189 (1996).
[16] J. M. López, Phys. Rev. Lett. 83, 4594 (1999).

[17] J. J. Ramasco, J. M. López, and M. A. Rodríguez, Phys. Rev. Lett. 84, 2199 (2000).

[18] M. Dubé, M. Rost, K. R. Elder, M. Alava, S. Majaniemi, and T. Ala-Nissila, Phys. Rev. Lett. 83, 1628 (1999).

[19] M. Dubé, M. Rost, and M. Alava, Eur. Phys. J. B 15, 691 (2000); 15, 701 (2000).

[20] A. Hernández-Machado, J. Soriano, A. M. Lacasta, M. A. Rodríguez, L. Ramírez-Piscina, and J. Ortín, Europhys. Lett. 55, 194 (2001).

[21] K. R. Elder, M. Grant, N. Provatas, and J. M. Kosterlitz, Phys. Rev. E 64, 021604 (2001).

[22] T. Laurila, C. Tong, I. Huopaniemi, S. Majaniemi, and T. AlaNissila, Eur. Phys. J. B 46, 553 (2005).

[23] K. Luo, M.-P Kuittu, C. Tong, S. Majaniemi, and T. AlaNissila, J. Chem. Phys. 123, 194702 (2005).

[24] P. C. Hohenberg and B. I. Halperin, Rev. Mod. Phys. 49, 435 (1977).

[25] E. W. Washburn, Phys. Rev. 17, 273 (1921).

[26] E. Pauné and J. Casademunt, Phys. Rev. Lett. 90, 144504 (2003).

[27] T. Laurila, C. Tong, I. Huopaniemi, S. Majaniemi, and T. AlaNissila, e-print cond-mat/0601473.

[28] A. Hernández-Machado, A. M. Lacasta, E. Mayoral, and E. Corvera-Poiré, Phys. Rev. E 68, 046310 (2003).

[29] J. M. López and M. A. Rodríguez, Phys. Rev. E 52, 6442 (1995).

[30] G. Parisi, Europhys. Lett. 17, 673 (1992). 